\title{
A preliminary study of transparency with chromatic colors
}

\author{
SERGIO CESARE MASIN \\ University of Udine, Udine, Italy \\ and \\ BAINGIO PINNA \\ University of Padua, Padua, Italy
}

\begin{abstract}
We studied the perception of transparency of chromatic surfaces within five unidimensional color continua in two experiments. In the first experiment, we tested a model for the perception of the density of a transparent surface. The results suggested that judgment of the density in any of the five unidimensional color continua was the composite outcome of the two opposite tendencies to judge background visibility at lower densities, and to judge surface salience at higher densities. In the second experiment, we tested Metelli's conditions for the occurrence of the kind of transparency that involves the perception of density. The results showed that these conditions were valid in all five unidimensional color continua.
\end{abstract}

Theoretical studies of transparency to date have focused on the perception of the transparency of achromatic surfaces on achromatic backgrounds (Beck, Prazdny, \& Ivry, 1984; Masin, 1984; Masin \& Gardonio, 1985; Metelli, $1974,1985)$. In this paper, we address the problem of the perception of transparency with both chromatic and achromatic colors.

Metelli (1985) proposed a model of achromatic transparency that encoded the conditions for the occurrence of the perception of transparency. Figure 1a represents schematically a rectangular achromatic surface transparent over a two-part achromatic background. Let $a, b, p$, and $q$ represent the achromatic colors of regions $\mathrm{A}, \mathrm{B}$, $P$, and $Q$, respectively, in the pattern illustrated in Figure 1a. Metelli proposed that $p$ and $q$ were given by the following averaging models (when the degree of transparency and the achromatic color of the transparent surface were the same on the two parts of the background):

and

$$
p=x a+(1-x) t
$$

$$
q=x b+(1-x) t
$$

where $t$ was interpreted as the achromatic color, and $x$ as the degree of transparency, of the transparent surface. According to Metelli, all the variables $(a, b, p, q, t$, and $x$ ) were assumed to vary in the interval $[0,1]$, where 0 and 1 mean, respectively "black" and "white" for $a$, $b, p, q$, and $t$, and "opacity" and "perfect transparency" for $x$. The following equation for $x$ may be derived from Models 1 and 2:

$$
x=(p-q) /(a-b)
$$

This study was supported by a grant from CNR to the first author (Bilateral Project with Japan). Mail should be directed to S. C. Masin, University of Udine, Institute of Philosophy, Pedagogics, and Teaching of Modern Languages, Via Antonini 8, 33100 Udine, Italy.
Equation 3 encodes two basic color conditions for the occurrence of the transparency phenomenon. Since $x$ varies in the interval $[0,1]$, for transparency to occur two conditions must be present: (I) the differences $p-q$ and $a-b$ must have the same sign, and (II) $|p-q|$ must be smaller than $|a-b|$. These conditions will be called Metelli's conditions for the occurrence of transparency.

Masin (1984) proposed a phenomenal model of transparency that encoded the achromatic color relations for the perception of the density (the opposite of the degree of transparency) of a transparent surface. This model, which was derived from experimental data, states that the degree of density depends on the differences in achromatic color between the parts inside the transparent surface and between the transparent surface and the parts of the background. In a pattern like the one in Figure 2, in which the outer contour depicts the edge of a square filter at a distance in front of a small square, the degree of density, $y$, of the filter was shown to be given by the following weighted average:

$$
y=w|l-m|+(1-w)(\mathrm{k}-|m-n|),
$$

where $l, m$, and $n$ are the achromatic colors, respectively, of parts $L, M$, and $N$, and $w$ is a weight coefficient. Each of the variables $l, m, n$, and $y$ is assumed to be rated using numbers in the interval $[0, k]$. Masin and Gardonio (1985) extended Model 4 to a pattern like the one depicted in Figure 1a and found data in favor of the following model:

$y=w_{1}|a-p|+w_{2}|q-b|+\left(1-w_{1}-w_{2}\right)(k-|p-q|)$,

where $w_{1}$ and $w_{2}$ are weight coefficients. Unfortunately, Models 4 and 5 allow only qualitative predictions because the weight coefficients are unknown and may vary with the differences in achromatic color (Masin, 1984). 

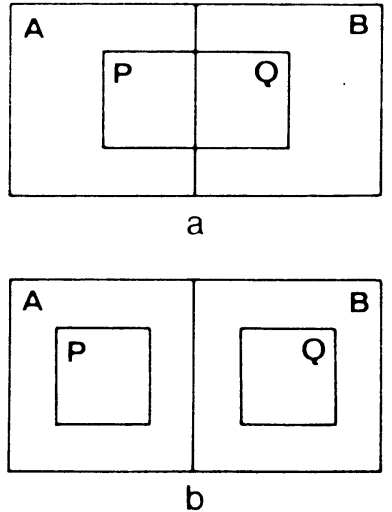

Figure 1. Experimental patterns used in Experiment 1. The letters $A, B, P$, and $Q$ indicate the parts of the pattern.

Phenomenological observation suggests that the following five unidimensional color continua may be distinguished: black-white, and (for a given value of chromaticness and blackness) yellow-red, red-blue, blue-green, and green-yellow.

The experiments reported here had two purposes. The first purpose (Experiment 1) was to study the nature of the relation between density, $y$, and the differences $a-p$, $p-q$, and $q-b$ in Model 5 , when $a, b, p$, and $q$ were chromatic colors within one unidimensional color continuum. In the literature (Beck et al., 1984; Metelli, 1985; Metelli, Masin, \& Manganelli, 1980), there is agreement that chromatic color scissioning in transparency may derive from a separation of two color components in regions $P$ and $Q$ (Figure 1a). We therefore assumed that the differences $a-p$ and $q-b$ corresponded to the proportions of $a$ in $p$, and of $b$ in $q$, respectively, whereas the difference $p-q$ corresponded to the differences between the proportions of $a$ and $b$ in both $p$ and $q$. The second purpose (Experiment 2) was to determine whether Metelli's conditions for the occurrence of transparency held in all five unidimensional color continua.

\section{EXPERIMENT 1}

\section{Method}

Observers. The observers were 40 university students at the University of Padua. Their color vision was checked for normality by the Ishihara test.

L

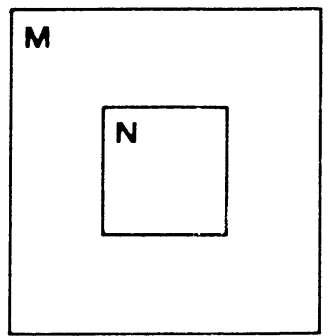

Figure 2. Proximal stimulus resulting from a square filter in front of a small square. The letters $L, M$, and $N$ indicate the parts of the stimulus.
Stimuli. The stimuli were patterns like the ones depicted in Figures 1a and $1 \mathrm{~b}$. Regions A, B, P, and Q were made by cutting out square pieces of paper from NCS color papers (Color Institute, Stockholm, Sweden). The following papers were used: Y, Y20R, Y50R, Y80R, and $\mathrm{R}$, all with the same blackness $(s=10)$ and chromaticness $(c=80)$; G, G20Y, G50Y, G80Y, and Y, all with $s=10$ and $c=70$; B, B20G, B50G, B80G, and G, all with $s=20$ and $c=60 ; \mathrm{R}, \mathrm{R} 20 \mathrm{~B}, \mathrm{R} 40 \mathrm{~B}, \mathrm{R} 60 \mathrm{~B}$, and $B$, all with $s=20$ and $c=50$; and pure gray papers with reflectance $.08, .16, .26, .40$, or .59 (the symbols are those used by the Swedish Standards Institution, 1984; capital letters are the initials of the name of a fundamental color; alphanumerical symbols represent a mixed color, with the number meaning the proportion of the color represented by the second letter in the alphanumerical symbol). The side length of squares corresponding to $\mathrm{A}$ and $\mathrm{B}$ and to $\mathrm{P}$ and $\mathrm{Q}$ was 40 and $22 \mathrm{~mm}$, respectively. Each pattern was stuck on a white $90 \times 125 \mathrm{~mm}$ cardboard rectangle having a reflectance of about .95 .

Each pattern was viewed from a distance of $1.5 \mathrm{~m}$ and was kept at eye level by the use of a head- and chinrest mounted on a table covered with black cardboard. The patterns were placed on a cardboard holder in the frontal parallel plane while they were covered with a screen made of the same black cardboard as the holder (reflectance .05). The patterns were displayed for 5-10 sec by removing the screen. The walls of the experimental room were covered with black velvet. The stimuli were illuminated by diffused light (Philips SL-13 lamps). The illumination level was of about $15 \mathrm{~lx}$.

Twenty-five patterns similar to that in Figure 1a, and 25 similar to that in Figure $1 \mathrm{~b}$, were made. Each of these two sets of patterns contained five subsets of five patterns. The colors of A and B did not vary in a subset. The symbols for the papers, or the reflectances, corresponding to the colors of $\mathrm{A}, \mathrm{B}, \mathrm{P}$, and $\mathrm{Q}$ in Figures $1 \mathrm{a}$ or $1 \mathrm{~b}$ are listed in Table 1 . The color of the rectangular transparent surfaces in patterns like the one in Figure 1a was gray, orange, green-yellow, blue-green, and purple in Patterns 1-5, 6-10, 11-15, 16-20, and 21-25, respectively.

As may be seen, the values chosen for $p$ and $q$ in all five unidimensional continua were such that $a<p<q<b$ (thus respecting Metelli's conditions for the occurrence of transparency), or $a=p$ and $q=b$, or $p=q$. The equality $p=q$ was approximate due to simultaneous contrast.

Procedure. The experiment was divided into two parts. In one part, the observers were asked to rate the degree of density of the rectangular transparent surface in patterns like the one in Figure 1a. The term "density" was defined to the observers by means of an achromatic photograph of seven filters varying in density (taken from Gyulai, 1976). It was pointed out to the observers that the greater the density the less the visibility of the part of the background seen through the filter. Density was rated using the numbers in the range from 0 to 100 , where 0 meant perfect transparency (as in a window pane) and 100 , opacity. ${ }^{1}$

In the other part of the experiment, the observers were asked to report the percentage of $a$ in $p$, of $a$ in $q$, of $b$ in $p$, and of $b$ in $q$ in patterns like the one in Figure 1b. The four judgments were produced in random order for each pattern. As an introduction to the color continua, the observer was shown the examples of the five color continua reported in the SSI (1984, Sheets 2 and 43) color atlas.

The observers were divided into two groups of 20 . One group first rated density and then reported the color percentage; for the other group this order was reversed. Half of the observers of each group had patterns with region $A$ on the left, and the other half had region $A$ on the right. The patterns were shown in random order twice.

\section{Results and Discussion}

The mean estimated percentages of $a$ and $b$ in $p$ and $q$ in patterns like that in Figure $1 b$, and the mean rated density in the corresponding patterns represented by Figure 1a, are reported in Table 2 . As may be seen, there was no substantial difference in the results among the five subsets of patterns. Figure 3 shows the results averaged over the five subsets. The ordered symbols P1 through P5, which are reported the same distances apart on the abscissa, represent the patterns in any one of the ordered sets of Patterns 1-5, 6-10, 11-15, 16-20, or 21-25. The mean estimates of density, $y$, and of the differences 
$|a-p|,|q-b|$, and $100-|p-q|$, are represented on the ordinate. The differences $|a-p|$ and $|q-b|$ were assumed to correspond to the differences between 100 and the mean estimate of the percentages of $a$ in $p$ and of $b$ in $q$, respectively. The difference $|p-q|$ was assumed to correspond to the average of the following two differences: between the mean percentages of $a$ in $p$ and $q$, and between the mean percentages of $b$ in $p$ and $q$. As may be seen after an easy calculation on the data in Table 2, these differences were substantially equal. The standard errors (computed using the observers' two estimates per pattern as scores) were smaller than the size of the symbols that represent the corresponding means in Figure 3 .

The weights in Model 5 have been shown to vary with the differences in achromatic color (Masin, 1984). This means that the magnitude of the effect on density produced by a given color difference depends on the difference value itself. The results in Figure 3 provide a hint as to the nature of the differential effects that color differences may have on the perception of density. Metelli (1975) found that observers reported that a given transparent surface with very low density was more transparent than another surface with high density because the background was more visible when the density was very low. However, the same observers described transparent surfaces as more salient at high densities, and thus sometimes "mistakenly" reported denser surfaces as being more transparent only because they were more salient. These findings suggest that there are two opposite tendencies in the judgment of density: at low densities, the observer heeds the background (and consequently judges density in terms of the degree

Table 1

Chromatic Color (Symbol of NCS Paper) or Reflectance of the Regions in Figures 1a and $1 b$

\begin{tabular}{|c|c|c|c|c|}
\hline \multirow{2}{*}{$\begin{array}{l}\text { Pattern } \\
\text { Number }\end{array}$} & \multicolumn{4}{|c|}{ (Experiment 1$)$} \\
\hline & A & $\mathbf{P}$ & Q & $\mathbf{B}$ \\
\hline 1 & .59 & .59 & .08 & .08 \\
\hline 2 & .59 & .40 & .16 & .08 \\
\hline 3 & .59 & .26 & .16 & .08 \\
\hline 4 & .59 & .40 & .26 & .08 \\
\hline 5 & .59 & .26 & .26 & .08 \\
\hline 6 & $\mathbf{R}$ & $\mathbf{R}$ & $\mathbf{Y}$ & $\mathbf{Y}$ \\
\hline 7 & $\mathbf{R}$ & Y80R & Y20R & $\mathbf{Y}$ \\
\hline 8 & $\mathbf{R}$ & Y50R & Y20R & $\mathbf{Y}$ \\
\hline 9 & $\mathbf{R}$ & Y80R & Y50R & $\mathbf{Y}$ \\
\hline 10 & $\mathbf{R}$ & Y50R & Y50R & $Y$ \\
\hline 11 & $Y$ & $\mathbf{Y}$ & G & G \\
\hline 12 & $Y$ & G80Y & G20Y & G \\
\hline 13 & $Y$ & G50Y & G20Y & G \\
\hline 14 & $\mathrm{Y}$ & G80Y & G50Y & G \\
\hline 15 & $\mathrm{Y}$ & G50Y & G50Y & G \\
\hline 16 & G & G & B & B \\
\hline 17 & $\mathbf{G}$ & B80G & B20G & B \\
\hline 18 & G & B50G & B20G & B \\
\hline 19 & $\mathbf{G}$ & B80G & B50G & B \\
\hline 20 & $\mathbf{G}$ & B50G & B50G & B \\
\hline 21 & B & B & $\mathbf{R}$ & $\mathbf{R}$ \\
\hline 22 & B & R60B & R20B & $\mathbf{R}$ \\
\hline 23 & B & R40B & R20B & $\mathbf{R}$ \\
\hline 24 & B & R60B & R20B & $\mathbf{R}$ \\
\hline 25 & B & R40B & R40B & $\mathbf{R}$ \\
\hline
\end{tabular}

Table 2

Mean Estimated Percentage of $a$ and $b$ in $p$ and $q$ (Figure 1b) and Mean Rated Density (Figure 1a) Obtained in Experiment 1

\begin{tabular}{cccccr}
\hline $\begin{array}{c}\text { Pattern } \\
\text { Number }\end{array}$ & \% $a$ in $p$ & \% $a$ in $q$ & $\% b$ in $q$ & $\% b$ in $p$ & $\begin{array}{r}\text { Rated } \\
\text { Density }\end{array}$ \\
\hline 1 & 99.9 & 4.0 & 99.9 & 4.6 & 0.0 \\
2 & 78.3 & 18.5 & 77.1 & 20.8 & 19.2 \\
3 & 78.3 & 56.9 & 42.4 & 20.3 & 58.3 \\
4 & 41.8 & 20.4 & 77.1 & 58.9 & 52.7 \\
5 & 43.1 & 58.8 & 42.5 & 58.4 & 100.0 \\
6 & 99.9 & 3.9 & 99.9 & 4.4 & 0.0 \\
7 & 78.1 & 19.4 & 76.4 & 19.7 & 20.8 \\
8 & 77.3 & 58.7 & 40.7 & 19.9 & 59.5 \\
9 & 41.1 & 20.4 & 76.5 & 60.1 & 54.4 \\
10 & 40.4 & 59.7 & 42.9 & 58.9 & 100.0 \\
11 & 100.0 & 3.9 & 100.0 & 3.3 & 0.0 \\
12 & 76.3 & 19.4 & 76.6 & 20.6 & 21.0 \\
13 & 77.5 & 57.9 & 40.8 & 23.6 & 60.5 \\
14 & 40.4 & 20.8 & 76.6 & 58.9 & 54.8 \\
15 & 41.0 & 60.6 & 42.0 & 59.3 & 100.0 \\
16 & 100.0 & 4.2 & 99.9 & 3.6 & 0.0 \\
17 & 78.3 & 19.9 & 77.4 & 20.1 & 23.1 \\
18 & 78.1 & 57.7 & 39.6 & 20.2 & 74.1 \\
19 & 40.8 & 21.3 & 75.9 & 59.3 & 54.1 \\
20 & 40.8 & 59.3 & 39.1 & 59.5 & 100.0 \\
21 & 99.9 & 4.5 & 99.7 & 4.1 & 0.0 \\
22 & 76.4 & 21.2 & 76.5 & 20.6 & 23.8 \\
23 & 77.7 & 57.0 & 40.0 & 20.8 & 63.3 \\
24 & 41.7 & 21.8 & 76.3 & 59.6 & 57.0 \\
25 & 40.9 & 59.6 & 39.9 & 59.6 & 99.8 \\
\hline & & & & &
\end{tabular}

of background visibility), and at high densities the observer heeds the transparent surface (and consequently judges density in terms of the degree of surface salience). The results reported in Figure 3 indicate which color differences may trigger these tendencies. Patterns $\mathrm{P} 1$ and $\mathrm{P} 2$ show that density is almost exclusively dependent on $|a-p|$ and $|q-b|$ (the difference between rated density and $100-|p-q|$ in Patterns $P 1$ and $P 2$ is significant well beyond the 0.001 level, $z$-test). The results for Patterns P3 and P4 show a composite effect of all three differences on density. The results for Pattern P5 show that the rated density is practically equal to 100 , even if all color differences are less than 100. In Pattern P5, parts P and Q were made with the same paper. However, the difference $p-q$ had a sign opposite of that of the difference $a-b$ (a violation of Metelli's Condition I) due to the simultaneous contrast of $b$ on $p$ and of $a$ on $q$. The observers accordingly reported opacity in this case. The results for Patterns P1-P4 suggest, therefore, that the visibility of the background depends on $|a-p|$ and $|b-q|$ at low densities, whereas the salience of the surface is plausibly affected by $|p-q|$ at high densities. The overall judgment of the degree of density would be, in general, a compromise between the two competing tendencies to judge the degrees of background visibility and of surface salience.

\section{EXPERIMENT 2}

\section{Method}

Observers. The observers were the same 40 used in Experiment 1. Stimuli. The stimuli had the same shape as that of the pattern in Figure 1a, and had the same sizes and were shown in the same illumi- 


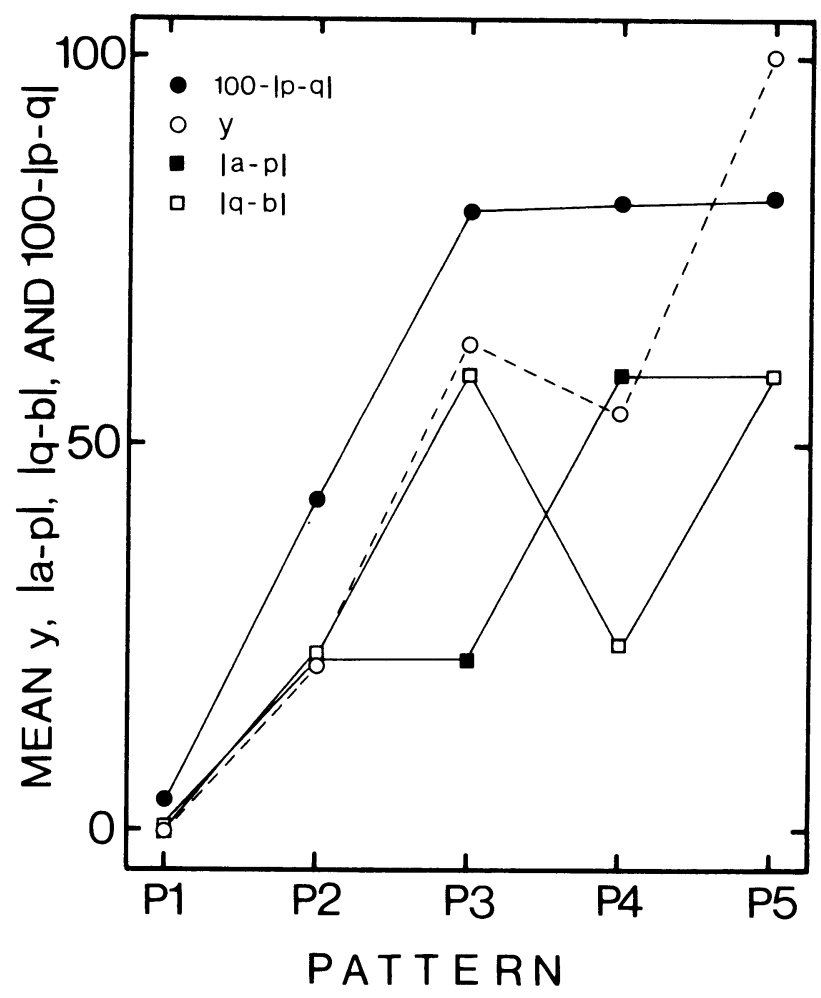

Figure 3. Representation of the mean estimated differences $|a-p|$, $|q-b|$, and $100-|p-q|$, and the mean rated density, $y$, as a function of Patterns P1-P5.

nation conditions as those used in Experiment 1 . Table 3 lists the symbols and the reflectances of the NCS papers used for the stimuli. As may be seen, Patterns 1-5 are Patterns 4, 9, 14, 19, and 24 listed in Table 1, whereas Patterns 6-10 were derived from Patterns 1-5 by exchanging the colors between $P$ and $Q$ (a violation of Metelli's Condition I), and Patterns 11-15 were derived from Patterns 1-5 by exchanging the colors between $A$ and $P$ and between $Q$ and $B$ (a violation of Metelli's Condition II).

Procedure. Each pattern was shown to the observer once in random order. The observer was asked to report whether or not there was trans-

Table 3

Stimuli Used in Experiment 2 and Frequency of Transparency Judgments

\begin{tabular}{cccccc}
\hline & \multicolumn{5}{c}{$\begin{array}{c}\text { Chromatic Color (Symbol of NCS Paper) or } \\
\text { Reflectance of the Regions in Figure 1a }\end{array}$} \\
\cline { 2 - 6 } Pattern & A & P & Q & B & Frequency \\
\hline 1 & .59 & .40 & .26 & .08 & 40 \\
2 & R & Y80R & Y50R & Y & 40 \\
3 & Y & G80Y & G50Y & G & 40 \\
4 & G & B80G & B50G & B & 40 \\
5 & B & R60B & R20B & R & 40 \\
6 & .59 & .26 & .40 & .08 & 0 \\
7 & R & Y50R & Y80R & Y & 0 \\
8 & Y & G50Y & G80Y & G & 0 \\
9 & G & B50G & B80G & B & 0 \\
10 & B & R20B & R60B & R & 0 \\
11 & .40 & .59 & .08 & .26 & 0 \\
12 & Y80R & R & Y & Y50R & 0 \\
13 & G80Y & Y & G & G50Y & 1 \\
14 & B80G & G & B & B50G & 17 \\
15 & R60B & B & R & R20B & 8 \\
\hline
\end{tabular}

parency in the pattern, while keeping in mind that if a surface was transparent it also had to have some density.

\section{Results}

The results of this experiment are reported in Table 3, in the rightmost column. As was to be expected from Experiment 1, all observers perceived transparency in Patterns 1-5. Metelli's Condition I was confirmed in all unidimensional color continua since no perception of transparency was reported for Patterns 6-10. One, 17, and 8 perceptions of transparency were reported for Patterns 13, 14, and 15, respectively, in which Metelli's Condition II was violated. However, in these cases, the observers reported that the transparency they perceived was different in kind from the transparency that occurred in Patterns 1-5. They described the special kind of transparent surface as "a sort of rectangle of light," or "a rectangle giving an impression of neatness or clearness." The rectangle was not reported to have a density. This special kind of transparency probably corresponded to the kind of transparency without scission of colors observed by Metzger (1955). ${ }^{2}$ We may therefore conclude that Metelli's Conditions I and II for the occurrence of the kind of transparency that involves the perception of density are confirmed in all unidimensional color continua.

\section{REFERENCES}

Beck, J., Prazdny, K., \& Ivry, R. (1984). The perception of transparency with achromatic colors. Perception \& Psychophysics, 35, 407-422.

GYULAI, E. (1976). Grado, colore e uniformità della transparenza fenomenica. Atti dell'Istituto Veneto di Scienze, Lettere ed Arti, 134, 791-803.

MAsin, S. C. (1984). An experimental comparison of three- versus foursurface phenomenal transparency. Perception \& Psychophysics, 35, 325-332.

MASIN, S. C., \& GARDonio, G. (1985). The valuation of the apparent density of a filter on a bicolored background. Perception \& Psychophysics, 37, 103-108.

METELLI, F. (1974). The perception of transparency. Scientific American, 230, 90-98.

Metelli, F. (1975). What does "more transparent" mean? A paradox. In M. Henle (Ed.), Vision and artifact (pp. 19-24). New York: Springer.

Metelli, F. (1985). Stimulation and perception of transparency. Psychological Research, 47, 185-202.

Metelli, F., Masin, S. C., \& Manganelli, A. M. (1980). Partial transparency. Atti dell'Accademia Patavina di Scienze, Lettere ed Arti, 92, 115-169.

METZGER, W. (1955). Über Durchsichtigkeitserscheinungen. Rivista di Psicologia, 49, 187-189.

SSI (1984). Colour atlas. Stockholm: Swedish Standards Institute.

\section{NOTES}

1. Before the execution of the experiment there was no proof that transparency occurred in all chromatic color continua when Metelli's conditions were respected. Therefore, the rating of density served first of all as a test of the occurrence of transparency when these conditions were respected.

2. One plausible reason why a Metzger's kind of transparency occurred in the blue-green and red-blue continua is that $a$ and $b$ were lighter than $p$ and $q$, even if $a, b, p$, and $q$ were matched in blackness and chromaticness on the basis of the SSI Colour Atlas.

(Manuscript received February 13, 1989.) 